\title{
Exponential Stability Criteria for Uncertain Stochastic Systems*
}

\author{
Li Yumei ${ }^{1,2}$, Guan Xinping ${ }^{1}$, Peng Dan ${ }^{1}$, Luo Xiaoyuan $^{1}$ \\ 1. Institute of Electrical Engineering Yanshan University, Qinhuangdao 066004, P. R. China \\ E-mail: xpguan@ysu.edu.cn \\ 2. Department of Mathematics and System Science, Xinjiang University, Urumuqi 830046, P. R. China \\ E-mail: zwzlym@163.com
}

\begin{abstract}
This paper considers the problem of delay-dependent exponential stability in mean square for a class of stochastic systems with polytopic-type uncertainties and time-varying delay. Based on the application of the descriptor model transformation and free weighting matrices to express this relationship among the system variables and among the terms in the Newton-Leibniz formula, some new delay-dependent exponential stability criteria are obtained in terms of linear matrix inequalities(LMIs). The new criteria are less conservative than existing ones. Numerical examples demonstrate the new criteria are effective and are an improvement over existing ones.
\end{abstract}

Key Words: Linear stochastic system, Exponential stability in mean square, Time-varying state delay, Delay-dependent criteria, Linear matrix inequality (LMI)

\section{INTRODUCTION}

Recently, delay-dependent stability criteria for stochastic systems with delay and system models expressed by Itôtype stochastic differential equations has gained extensive attention (see [1-8]). In view of the robustness of stochastic stability, the linear and semi-linear systems are studied in [1] and [2], respectively. [3] investigated the stability of linear and semi-linear stochastic differential equation by means of the exponential stability. Verriest [4] presented stability of linear stochastic differential equation via Riccati equations. Based on the LMI approach, [5-8] gave the delay-dependent robust stability criteria of uncertain stochastic systems, respectively. However, the criteria in [5] involved the parameterized model transformation, to determine the stability of the system, [6] and [8] used some inequality constraint, [7] used a descriptor integral inequality constraint, and the criteria in [7] and [8] with matrix constraint $P \leqslant \alpha I,(\alpha>0$ is a scalar, $P$ is the product of Lyapunov matrix ), these results show considerable conservativeness.

This paper presents some new delay-dependent exponential stability criteria for continuous-time linear stochastic system with polytopic-type uncertainties and time-varying delay. First, by extending the descriptor system approach introduced in [9] for deterministic systems to the stochastic systems case. We represent the stochastic systems in the equivalent descriptor stochastic systems. Based on the equivalent descriptor form representation, we choose a new type of Lyapunov-Krasovskii functional. Second, in order to exclude those constraint conditions in [6-8], some free weighting matrices introduced into the proof. Finally, based on LMI algorithm we respectively obtain a less conservative delay-dependent exponential stability criteria for stochastic system with polytopic-type uncertainties and time-varying delay . Numerical simulation examples show the results are effective and are an improvement over existing criteria.

For convenience, we adopt the following notations: $\operatorname{Tr}(A)\left(A^{\mathrm{T}}\right): \quad$ Trace (transpose) of the matrix $A$.

${ }^{*}$ This work was supported in part by NSFC under Grant 60704009 and NSF of Hebei Province P.R. China under Grant F2005000390, F2006000270.
$A \geqslant 0(A>0): \quad$ Positive semi-definite (positive definite) matrix $A$.

$I$ : Identity matrix.

$\|x\|:=\left(\sum_{i=1}^{n} x_{i}^{2}\right)^{\frac{1}{2}}: \quad$ Euclidean norm in $R^{n}$.

$L_{\digamma_{0}}^{2}\left([-\tau, 0] ; R^{n}\right)$ : the family of $R^{n}$-valued stochastic processes $\eta(s),-\tau \leqslant s \leqslant 0$ such that $\eta(s)$ is $\digamma_{0}-$ measurable for every second and $\int_{-\tau}^{0} E\|\eta(s)\|^{2} \mathrm{~d} s<\infty$

$E\{\cdot\}$ : mathematical expectation operator with respect to the given probability measure $P$.

\section{PRELIMINARIES}

Consider the robust stability of system (1) with polytopictype uncertainties, that is, assuming that the system (1) have the following form:

$$
\begin{aligned}
\mathrm{d} x(t)= & {\left[A x(t)+A_{d} x(t-h(t))\right] \mathrm{d} t } \\
& +\left[C x(t)+C_{d} x(t-h(t))\right] \mathrm{d} \beta(t) \\
x(t)= & \varphi(t), \forall t \in[-\tau, 0]
\end{aligned}
$$

where $x(t) \in R^{n}$ is the state vector, the system matrices $A, A_{d}, C$ and $C_{d}$ are assumed to be uncertain but belonging to a known convex compact set of polytopic type, namely

$$
\left(A, A_{d}, C, C_{d}\right) \in \Omega
$$

where $\Omega$ is a given convex bounded polyhedral domain described by $q$ vertices as follows:

$$
\begin{gathered}
\Omega:=\left\{\left(A, A_{d}, C, C_{d}\right)=\sum_{k=1}^{q} \xi_{k}\left(A_{k}, A_{k d}, C_{k}, C_{k d}\right)\right. \\
\left.\xi_{k} \geqslant 0 ; \sum_{k=1}^{q} \xi_{k}=1\right\}
\end{gathered}
$$

The time delay $h(t)$ is a time-varying continuous function that satisfies

$$
0 \leqslant h(t) \leqslant \tau
$$

and

$$
\dot{h(t)} \leqslant \mu \leqslant 1
$$


where $\tau$ and $\mu$ are constants and $\varphi(t)$ is a continuous vectorvalued initial function. and $\varphi:=\{\varphi(s):-\tau \leqslant s \leqslant 0\} \in$ $L_{\digamma_{0}}^{2}\left([-\tau, 0], R^{n}\right)$ It is well known that (1) has an unique solution, denoted by $x(t, \varphi)$, which is square integrable. So (1) admits a trivial solution $x(t, 0) \equiv 0 . A_{k}, A_{k d}, C_{k}, C_{k d}$ are known constant matrices with appropriate dimensions. the variables $\beta(t)$ are an m-dimensional Brownian motion defined on a complete probability space $(\Omega, \digamma, P)$ with a natural filtration $\left\{\digamma_{t}\right\}_{t \geqslant 0}$ (i.e.., $\left.\digamma_{t}=\sigma\{\varpi(s): 0 \leqslant s \leqslant t\}\right)$.

Definition System (1) is said to be exponentially stable in mean square if there exists a positive constant $\alpha_{0}$ such that

$$
\lim _{t \rightarrow \infty} \sup \frac{1}{t} \log E\|x(t)\|^{2} \leqslant-\alpha_{0}
$$

\section{DELAY-DEPENDENT ROBUST EXPONEN- TIAL STABILITY}

In order to discuss the stability of system (1), first, we introduce the descriptor system approach, set

$$
\begin{aligned}
& q(t)=A x(t)+A_{d} x(t-h(t)) \\
& g(t)=C x(t)+C_{d} x(t-h(t))
\end{aligned}
$$

then system (1) becomes the following descriptor stochastic systems

$$
d x(t)=q(t) d t+g(t) d \beta(t)
$$

Moreover, equations in (5), (6) ensure that the following zero equations

$$
\begin{gathered}
2\left[x^{\mathrm{T}}(t) N_{1}+x^{\mathrm{T}}(t-h(t)) N_{2}+q^{\mathrm{T}}(t) N_{3}+g^{\mathrm{T}}(t) N_{4}\right] \\
*\left[A_{0} x(t)+A_{d} x(t-h(t))-q(t)\right] \equiv 0 \\
2\left[x^{\mathrm{T}}(t) T_{1}+x^{\mathrm{T}}(t-h(t)) T_{2}+q^{\mathrm{T}}(t) T_{3}+g^{\mathrm{T}}(t) T_{4}\right] \\
*\left[C x(t)+C_{d} x(t-h(t))-g(t)\right] \equiv 0
\end{gathered}
$$

where $N_{r}$ and $T_{r}(r=1,2,3,4)$ are appropriately dimensioned matrices. On the other hand, the Newton-Leibniz formula provides

$$
\begin{aligned}
x(t)-x(t-h(t)) & =\int_{t-h(t)}^{T} \dot{x}(s) \mathrm{d} s \\
& =\int_{t-h(t)}^{T} q(s) \mathrm{d} s-\varsigma
\end{aligned}
$$

where $\varsigma^{\mathrm{T}}=\left[\int_{t-h(t)}^{T} g(s) d \beta(s)\right]^{\mathrm{T}}$, by (10), get

$$
\int_{t-h(t)}^{T} q(s) \mathrm{d} s=x(t)-x(t-h(t))-\varsigma
$$

then, we obtained the following Theorem.

Theorem Consider the system (1) with polytopic-type uncertainties (2) and a time-varying delay satisfying (3) and (4). Given scalars $\tau>0$, and $\mu<1$, system (1) is robust exponentially stable in mean square, if there exist symmetric positive definite matrix $P_{k}>0, Q_{k} \geqslant 0, Z_{k}>0, R_{i j}^{(k)}$ $(i, j=1,2,3)$, and appropriately dimensioned matrices
$N_{r}$ and $T_{r}(r=1,2,3,4)$ such that $R_{i j}^{(k)}=R_{j i}^{(k)}$ and the following LMI holds for $k=1, \cdots, q$ :

$$
\Phi^{(k)}=\left[\begin{array}{ccccc}
\Phi_{11}^{(k)} & \Phi_{12}^{(k)} & \Phi_{13}^{(k)} & \Phi_{14}^{(k)} & R_{13}^{(k)} \\
* & \Phi_{22}^{(k)} & \Phi_{23}^{(k)} & \Phi_{24}^{(k)} & R_{23}^{(k)} \\
* & * & \Phi_{33}^{(k)} & \Phi_{34}^{(k)} & 0 \\
* & * & * & \Phi_{44}^{(k)} & 0 \\
* & * & * & * & -Z_{k}
\end{array}\right]<0
$$

and

$$
R^{(k)}=\left[\begin{array}{ccc}
R_{11}^{(k)} & R_{12}^{(k)} & R_{13}^{(k)} \\
* & R_{22}^{(k)} & R_{23}^{(k)} \\
* & * & R_{33}^{(k)}
\end{array}\right]>0
$$

where an ellipsis $*$ denotes a block induced easily by symmetry, and

$$
\begin{aligned}
\Phi_{11}^{(k)}= & N_{1} A_{k}+A_{k}^{\mathrm{T}} N_{1}^{\mathrm{T}}+T_{1} C_{k}+C_{k}^{\mathrm{T}} T_{1}^{\mathrm{T}}+Q_{k}+\tau R_{11}^{(k)} \\
& +R_{13}^{(k)}+R_{13}^{(k) \mathrm{T}} \\
\Phi_{12}^{(k)}= & N_{1} A_{d k}+A_{k}^{\mathrm{T}} N_{2}^{\mathrm{T}}+T_{1} C_{d k}+C_{k}^{\mathrm{T}} T_{2}^{\mathrm{T}}+\tau R_{12}^{(k)} \\
& -R_{13}^{(k)}+R_{23}^{(k) \mathrm{T}} \\
\Phi_{13}^{(k)}= & P_{k}-N_{1}+A_{k}^{\mathrm{T}} N_{3}^{\mathrm{T}}+C_{k}^{\mathrm{T}} T_{3}^{\mathrm{T}} \\
\Phi_{14}^{(k)}= & A_{k}^{\mathrm{T}} N_{4}^{\mathrm{T}}-T_{1}+C_{k}^{\mathrm{T}} T_{4}^{\mathrm{T}} \\
\Phi_{22}^{(k)}= & N_{2} A_{d k}+A_{d k}^{\mathrm{T}} N_{2}^{\mathrm{T}}+T_{2} C_{d k}-(1-\mu) Q_{k} \\
& +C_{d k}^{\mathrm{T}} T_{2}^{\mathrm{T}}+\tau R_{22}^{(k)}-R_{23}^{(k)}-R_{23}^{(k) \mathrm{T}} \\
\Phi_{23}^{(k)}= & -N_{2}+A_{d k}^{\mathrm{T}} N_{3}^{\mathrm{T}}+C_{d k}^{\mathrm{T}} T_{3}^{\mathrm{T}} \\
\Phi_{24}^{(k)}= & A_{d k}^{\mathrm{T}} N_{4}^{\mathrm{T}}-T_{2}+C_{d k}^{\mathrm{T}} T_{4}^{\mathrm{T}} \\
\Phi_{33}^{(k)}= & -N_{3}-N_{3}^{\mathrm{T}}+\tau R_{33}^{(k)}, \Phi_{34}^{(k)}=-N_{4}^{\mathrm{T}}-T_{3} \\
\Phi_{44}^{(k)}= & P_{k}-T_{4}^{\mathrm{T}}-T_{4}+\tau Z_{k}
\end{aligned}
$$

Proof Choose a Lyapunov-Krasovskii functional for system (1) to be

$$
V(t)=\sum_{i=1}^{5} V_{i}(t)
$$

in which

$$
\begin{gathered}
V_{1}(t)=\sum_{k=1}^{q} x(t)^{T} P_{k} x(t), V_{2}(t)=\sum_{k=1}^{q} \int_{t-h(t)}^{T} x^{\mathrm{T}}(s) Q_{k} x(s) \mathrm{d} s \\
V_{3}(t)=\sum_{k=1}^{q} \int_{-\tau}^{0} \int_{t+\theta}^{T} q^{\mathrm{T}}(s) R_{33}^{(k)} q(s) \mathrm{d} s \mathrm{~d} \theta \\
V_{4}(t)=\sum_{k=1}^{q} \int_{-\tau}^{0} \int_{t+\theta}^{T} \operatorname{trace}\left[g^{\mathrm{T}}(s) Z_{k} g(s)\right] \mathrm{d} s \mathrm{~d} \theta \\
V_{5}(t)=\sum_{k=1}^{q} \int_{0}^{T} \int_{\alpha-h(\alpha)}^{\alpha} \delta R_{k} \delta^{\mathrm{T}} \mathrm{d} s \mathrm{~d} \alpha
\end{gathered}
$$

where $\delta^{\mathrm{T}}=\left[x^{\mathrm{T}}(\alpha), x^{\mathrm{T}}(\alpha-h(a)), q^{\mathrm{T}}(s)\right]$

$$
R^{(k)}=\left[\begin{array}{ccc}
R_{11}^{(k)} & R_{12}^{(k)} & R_{13}^{(k)} \\
* & R_{22}^{(k)} & R_{23}^{(k)} \\
* & * & R_{33}^{(k)}
\end{array}\right]
$$


$P_{k}, Q_{k}, Z_{k}, R_{i j}^{(k)}(i, j=1,2,3)$ are positive definite matrices with appropriate dimensions. $L$ be the weak infinitesimal operator of (7), then, by Itô differential formula

$$
\begin{aligned}
& L_{v=0} V_{1}=\sum_{k=1}^{q}\left\{2 x^{\mathrm{T}}(t) P_{k} q(t)+\operatorname{trace}\left[g^{\mathrm{T}}(t) P_{k} g(t)\right]\right\} \\
& L_{v=0} V_{2} \leqslant \sum_{k=1}^{q}\left\{x^{\mathrm{T}}(t) Q_{k} x(t)\right. \\
& \left.-(1-\mu) x^{\mathrm{T}}(t-h(t)) Q_{k} x(t-h(t))\right\} \\
& L_{v=0} V_{3} \leqslant \sum_{k=1}^{q}\left\{\tau q^{\mathrm{T}}(t) R_{33}^{(k)} q(t)\right. \\
& \left.-\int_{t-h(t)}^{T} q^{\mathrm{T}}(s) R_{33}^{(k)} q(s) \mathrm{d} s\right\} \\
& L_{v=0} V_{4} \leqslant \sum_{k=1}^{q}\left\{\text { rtrace }\left[g^{\mathrm{T}}(t) Z_{k} g(t)\right]\right. \\
& \left.-\int_{t-h(t)}^{T} \operatorname{trace}\left[g^{\mathrm{T}}(s) Z_{k} g(s)\right] \mathrm{d} s\right\} \\
& L_{v=0} V_{5}=\sum_{k=1}^{q}\left\{h(t) \xi^{\mathrm{T}}\left[\begin{array}{cc}
R_{11}^{(k)} & R_{12}^{(k)} \\
R_{12}^{(k) T} & R_{22}^{(k)}
\end{array}\right] \xi\right. \\
& +2 \int_{t-h(t)}^{T} \xi^{\mathrm{T}}\left[\begin{array}{c}
R_{13}^{(k)} \\
R_{23}^{(k)}
\end{array}\right] q(s) \mathrm{d} s \\
& \left.+\int_{t-h(t)}^{T} q^{\mathrm{T}}(s) R_{33}^{(k)} q(s) \mathrm{d} s\right\}
\end{aligned}
$$

Substitute (11) into (17), get

$$
\begin{aligned}
& L_{v=0} V_{5}=\sum_{k=1}^{q}\left\{h(t) \xi^{\mathrm{T}}\left[\begin{array}{cc}
R_{11}^{(k)} & R_{12}^{(k)} \\
R_{12}^{(k) T} & R_{22}^{(k)}
\end{array}\right] \xi\right. \\
& +2 \xi^{\mathrm{T}}\left[\begin{array}{c}
R_{13}^{(k)}-R_{13}^{(k)} \\
R_{23}^{(k)}-R_{23}^{(k)}
\end{array}\right] \xi+\int_{t-h(t)}^{T} q^{\mathrm{T}}(s) R_{33}^{(k)} q(s) \mathrm{d} s \\
& \left.-2 \xi^{\mathrm{T}}\left[\begin{array}{l}
R_{13}^{(k)} \\
R_{23}^{(k)}
\end{array}\right] \varsigma\right\}
\end{aligned}
$$

By Lemma[11], for any matrix $Z_{k}>0$

$$
\begin{gathered}
-2 \xi^{\mathrm{T}}\left[\begin{array}{c}
R_{13}^{(k)} \\
R_{23}^{(k)}
\end{array}\right] \varsigma \\
\leqslant \xi^{\mathrm{T}}\left[\begin{array}{c}
R_{13}^{(k)} \\
R_{23}^{(k)}
\end{array}\right] Z_{k}^{-1}\left[\begin{array}{c}
R_{13}^{(k)} \\
R_{23}^{(k)}
\end{array}\right]^{\mathrm{T}} \xi+\varsigma^{\mathrm{T}} Z_{k} \varsigma
\end{gathered}
$$

Obviously

$$
\begin{aligned}
& L_{v=0} V_{5} \leqslant \sum_{k=1}^{q}\left\{\tau \xi^{\mathrm{T}}\left[\begin{array}{cc}
R_{11}^{(k)} & R_{12}^{(k)} \\
R_{12}^{T(k)} & R_{22}^{(k)}
\end{array}\right] \xi\right. \\
& +2 \xi^{\mathrm{T}}\left[\begin{array}{c}
R_{13}^{(k)}-R_{13}^{(k)} \\
R_{23}^{(k)}-R_{23}^{(k)}
\end{array}\right] \xi+\int_{t-h(t)}^{T} q^{\mathrm{T}}(s) R_{33}^{(k)} q(s) \mathrm{d} s \\
& \left.+\varsigma^{\mathrm{T}} Z_{k} \varsigma+\xi^{\mathrm{T}}\left[\begin{array}{c}
R_{13}^{(k)} \\
R_{23}^{(k)}
\end{array}\right] Z_{k}^{-1}\left[\begin{array}{c}
R_{13}^{(k)} \\
R_{23}^{(k)}
\end{array}\right]^{\mathrm{T}} \xi\right\}
\end{aligned}
$$

where $\xi^{\mathrm{T}}=\left[x^{\mathrm{T}}(t), x^{\mathrm{T}}(t-h(t))\right]$.

Combining $L_{v=0} V_{i}(i=1,2,3,4,5)$ and adding the terms on the left of (8)-(9) to $L_{v=0} V$ allows us to express $L_{v=0} V$ as

$$
\begin{aligned}
L_{v=0} V \leqslant & \sum_{k=1}^{q}\left\{\eta^{\mathrm{T}}(t) \xi_{k} \Phi^{(k)} \eta(t)\right. \\
& \left.-\int_{t-h(t)}^{T} \operatorname{trace}\left[g^{\mathrm{T}}(s) Z_{k} g(s)\right] \mathrm{d} s+\varsigma^{\mathrm{T}} Z \varsigma\right\}
\end{aligned}
$$

where

$$
\begin{aligned}
\eta^{\mathrm{T}}(t)= & {\left[x^{\mathrm{T}}(t), x^{\mathrm{T}}(t-h(t)), q^{\mathrm{T}}(t), g^{\mathrm{T}}(t)\right] } \\
\Phi^{(k)}= & {\left[\begin{array}{cccc}
\Phi_{11}^{(k)} & \Phi_{12}^{(k)} & \Phi_{13}^{(k)} & \Phi_{14}^{(k)} \\
* & \Phi_{22}^{(k)} & \Phi_{23}^{(k)} & \Phi_{24}^{(k)} \\
* & * & \Phi_{33}^{(k)} & \Phi_{34}^{(k)} \\
* & * & * & \Phi_{44}^{(k)}
\end{array}\right]+\sigma Z_{k}^{-1} \sigma^{\mathrm{T}} }
\end{aligned}
$$

where $\sigma^{\mathrm{T}}=\left[\left(R_{13}^{(k)}\right)^{\mathrm{T}},\left(R_{23}^{(k)}\right)^{\mathrm{T}}, 0,0\right]$, Since

$$
E\left(\varsigma^{\mathrm{T}} Z_{k} \varsigma\right)=E \int_{t-h(t)}^{T} \operatorname{trace}\left[g^{\mathrm{T}}(s) Z_{k} g(s)\right] \mathrm{d} s
$$

It follows that

$$
E L_{v=0} V(t) \leqslant \sum_{k=1}^{q} E \eta^{\mathrm{T}}(t) \xi_{k} \Phi^{(k)} \eta(t)
$$

By Schur's complement, $\Phi^{(k)}<0$ is equivalent to LMI (12). Next to show the expected exponential mean-square stability of system (1) set $\lambda_{0 k}=\lambda_{\min }\left(-\xi_{k} \Phi^{(k)}\right)$,

$$
\begin{gathered}
\lambda_{1 k}=\lambda_{\min }\left(P_{k}\right), \lambda=\sum_{k=1}^{q} \lambda_{0 k}, \alpha_{3}=\sum_{k=1}^{q} \lambda_{1 k} \text { by (20) } \\
E L_{v=0} V(t) \leqslant-\sum_{k=1}^{q} \lambda_{0 k} E \eta^{\mathrm{T}}(t) \eta(t) \leqslant-\lambda E x^{\mathrm{T}}(t) x(t)
\end{gathered}
$$

From the definitions of $V(t), q(t)$ and $g(t)$, there exist positive scalars $\alpha_{1}, \alpha_{2}$ such that

$$
\alpha_{3}\|x(t)\|^{2} \leqslant V(t) \leqslant \alpha_{1}\|x(t)\|^{2}+\alpha_{2} \int_{t-2 \tau}^{T}\|x(s)\|^{2} \mathrm{~d} s
$$

Choose a scalar $\alpha_{0}>0$ such that

$$
\alpha_{0}\left(\alpha_{1}+2 \alpha_{2} \tau e^{2 \alpha_{0} \tau}\right) \leqslant \lambda
$$

then, by Itô differential formula, for $t_{0} \geqslant 2 \tau$, it has

$$
\begin{aligned}
& E e^{\alpha_{0} t} V(t)-E e^{\alpha_{0} t_{0}} V\left(t_{0}\right)=E \int_{t_{0}}^{T} L_{v=0}\left(e^{\alpha_{0} s} V(s)\right) \mathrm{d} s \\
\leqslant & E \int_{t_{0}}^{T} e^{\alpha_{0} s}\left[\alpha _ { 0 } \left(\alpha_{1}\|x(s)\|^{2}\right.\right. \\
& \left.\left.+\alpha_{2} \int_{s-2 \tau}^{s}\|x(u)\|^{2} \mathrm{~d} u\right)-\lambda\|x(s)\|^{2}\right] \mathrm{d} s
\end{aligned}
$$


Note that

$$
\begin{aligned}
& \int_{t_{0}}^{T} e^{\alpha_{0} s} \int_{s-2 \tau}^{s}\|x(u)\|^{2} \mathrm{~d} u \mathrm{~d} s \\
\leqslant & \int_{t_{0}-2 \tau}^{T} \mathrm{~d} u \int_{u}^{u+2 \tau} e^{\alpha_{0} s}\|x(u)\|^{2} \mathrm{~d} s \\
\leqslant & 2 \tau e^{2 \alpha_{0} \tau} \int_{t_{0}}^{T} e^{\alpha_{0} s}\|x(s)\|^{2} \mathrm{~d} s+2 \tau e^{2 \alpha_{0} \tau} \int_{t_{0}-2 \tau}^{t_{0}}\|\xi(\theta)\| \mathrm{d} \theta
\end{aligned}
$$

By (21) and (22), getting

$$
\lim _{t \rightarrow \infty} \sup \frac{1}{t} \log E\|x(t)\|^{2} \leqslant-\alpha_{0}
$$

which implies system (1) is exponentially stable in mean square. The proof of Theorem 1 is completed.

Note that a delay-dependent and rate-independent exponential stability criterion for system (1) with polytopic-type uncertainties (2) and a delay satisfying (3) and (4) can be derived from Theorem 1 by choosing $Q_{k}=0$ as follows.

Corollary 1 Given scalar $\tau>0$, system (1) with polytopic-type uncertainties (2) and a time-varying delay satisfying (3) is exponentially stable in mean square if there exist symmetric positive-define $P_{k}, Z_{k}, R_{i j}^{(k)}(k=1, \cdots, q)$ and appropriately dimensioned matrices $N_{r}$ and $T_{r}(r=$ $1,2,3)$, Such that $R_{i j}^{(k)}=R_{j i}^{(k)}(i, j=1,2,3)$ and the following LMI holds for $k=1,2, \ldots, q$ :

$$
\hat{\Phi}^{(k)}=\left[\begin{array}{ccccc}
\hat{\Phi}_{11}^{(k)} & \Phi_{12}^{(k)} & \Phi_{13}^{(k)} & \Phi_{14}^{(k)} & R_{13}^{(k)} \\
* & \hat{\Phi}_{22}^{(k)} & \Phi_{23}^{(k)} & \Phi_{24}^{(k)} & R_{23}^{(k)} \\
* & * & \Phi_{33}^{(k)} & \Phi_{34}^{(k)} & 0 \\
* & * & * & \Phi_{44}^{(k)} & 0 \\
* & * & * & * & -Z_{k}
\end{array}\right]<0
$$

where

$$
\begin{aligned}
\hat{\Phi}_{11}^{(k)}= & N_{1} A_{k}+A_{k}^{\mathrm{T}} N_{1}^{\mathrm{T}}+T_{1} C_{k}+C_{k}^{\mathrm{T}} T_{1}^{\mathrm{T}} \\
& +\tau R_{11}^{(k)}+R_{13}^{(k)}+R_{13}^{(k) \mathrm{T}} \\
\hat{\Phi}_{22}^{(k)}= & N_{2} A_{d k}+A_{d k}^{\mathrm{T}} N_{2}^{\mathrm{T}}+T_{2} C_{d k} \\
& +C_{d k}^{\mathrm{T}} T_{2}^{\mathrm{T}}+\tau R_{22}^{(k)}-R_{23}^{(k)}-R_{23}^{(k) \mathrm{T}}
\end{aligned}
$$

and $\Phi_{i j}^{(k)}(i=1,2,3 ; j=2,3,4)$ are defined in (12).

In the case, when there is no stochastic uncertainty in the system (1), that is, $\beta(t)$ is assumed to be zero, system (1) degenerate into system [13]. Theorem 1 is specialized to the following corollary.

Corollary 2 Given scalars $\tau>0$, and $\mu<1$, system (1) with $\beta(t)=0$ and with time-varying delay satisfying (3) and (4) is asymptotically stable, if there exist symmetric positive-definite matrix $P_{k}>0, Q_{k} \geqslant 0, R_{i j}^{(k)}(i, j=1,2,3)$, and appropriately dimensioned matrices $N_{r}(r=1,2,3,4)$ such that $R_{i j}^{(k)}=R_{j i}^{(k)}$ and the following LMI holds for $k=1,2, \ldots, q$ :

$$
\Sigma^{(k)}=\left[\begin{array}{ccc}
\Sigma_{11}^{(k)} & \Sigma_{12}^{(k)} & \Sigma_{13}^{(k)} \\
* & \Sigma_{22}^{(k)} & \Sigma_{23}^{(k)} \\
* & * & \Sigma_{33}^{(k)}
\end{array}\right]<0
$$

and

$$
R^{(k)}=\left[\begin{array}{ccc}
R_{11}^{(k)} & R_{12}^{(k)} & R_{13}^{(k)} \\
* & R_{22}^{(k)} & R_{23}^{(k)} \\
* & * & R_{33}^{(k)}
\end{array}\right]>0
$$

where

$$
\begin{aligned}
\Sigma_{11}^{(k)}= & N_{1} A_{k}+A_{k}^{\mathrm{T}} N_{1}^{\mathrm{T}}+Q_{k}+\tau R_{11}^{(k)}+R_{13}^{(k)}+R_{13}^{(k) T} \\
\Sigma_{12}^{(k)}= & N_{1} A_{d k}+A_{k}^{\mathrm{T}} N_{2}^{\mathrm{T}}+\tau R_{12}^{(k)}-R_{13}^{(k)}+R_{23}^{(k) T} \\
\Sigma_{13}^{(k)}= & P_{k}-N_{1}+A_{k}^{\mathrm{T}} N_{3}^{\mathrm{T}} \\
\Sigma_{22}^{(k)}= & N_{2} A_{d k}+A_{d k}^{\mathrm{T}} N_{2}^{\mathrm{T}}-(1-\mu) Q_{k} \\
& +\tau R_{22}^{(k)}-R_{23}^{(k)}-R_{23}^{(k) T} \\
\Sigma_{23}^{(k)}= & -N_{2}+A_{d k}^{\mathrm{T}} N_{3}^{\mathrm{T}} \\
\Sigma_{33}^{(k)}= & -N_{3}-N_{3}^{\mathrm{T}}+\tau R_{33}^{(k)}
\end{aligned}
$$

Remark 1 Corollary 2 corresponds to Theorem $2^{[13]}$. Moreover, owing to we choose the different LyapunovKrasovskii functional, the results obtained from Corollary 2 are less conservation than existing ones ${ }^{[13]}$.

In addition, when we assume $A_{d k}=C_{d k}=0$,system (1) degenerates into a stochastic system without time-delay, Theorem 1 can be specialized to the following corollary.

Corollary 3 Given scalars $\tau>0$,and $\mu<1$, system (1) with time-varying delay satisfying (3) and (4) and with $A_{d k}=C_{d k}=0$ is exponentially stable in mean square, if there exist symmetric positive definite matrix $P_{k}>0$, and appropriately dimensioned matrices $N_{r}$ and $T_{r}(r=1,3,4)$ such that the following LMI holds for $k=1,2, \ldots, q$ :

$$
\hat{\Sigma}^{(k)}=\left[\begin{array}{ccc}
\hat{\Sigma}_{11}^{(k)} & \hat{\Sigma}_{13}^{(k)} & \hat{\Sigma}_{14}^{(k)} \\
* & \hat{\Sigma}_{33}^{(k)} & \breve{\Sigma}_{34}^{(k)} \\
* & * & \hat{\Sigma}_{44}^{(k)}
\end{array}\right]<0
$$

where

$$
\begin{aligned}
& \hat{\Sigma}_{11}^{(k)}=N_{1} A_{k}+A_{k}^{\mathrm{T}} N_{1}^{\mathrm{T}}+T_{1} C_{k}+C_{k}^{\mathrm{T}} T_{1}^{\mathrm{T}} \\
& \hat{\Sigma}_{13}^{(k)}=P_{k}-N_{1}+A_{k}^{\mathrm{T}} N_{3}^{\mathrm{T}}+C_{k}^{\mathrm{T}} T_{3}^{\mathrm{T}} \\
& \hat{\Sigma}_{14}^{(k)}=A_{k}^{\mathrm{T}} N_{4}^{\mathrm{T}}-T_{1}+C_{k}^{\mathrm{T}} T_{4}^{\mathrm{T}}, \hat{\Sigma}_{33}^{(k)}=-N_{3}-N_{3}^{\mathrm{T}} \\
& \hat{\Sigma}_{34}^{(k)}=-N_{4}^{\mathrm{T}}-T_{3}, \hat{\Sigma}_{44}^{(k)}=P_{k}-T_{4}^{\mathrm{T}}-T_{4}
\end{aligned}
$$

Remark 2 When $\mu=0$, the delay is time-invariant, from Theorem 1 we can easily obtain the delay-dependent exponential stability criteria for continuous-time linear stochastic system with polytopic-type uncertainties and with timeinvariant state delay.

\section{NUMERICAL SIMULATION}

In this section, for the purpose of illustrating the usefulness and flexibility of the developed in this paper, we present some simulation examples.

Example 1 Consider the following time-varying delay system $\Sigma_{1}$ with polytopic-type uncertainties ([13], Example 2): where

$$
A=\left[\begin{array}{cc}
0 & -0.12+12 \rho \\
1 & -0.465-\rho
\end{array}\right], A_{d}=\left[\begin{array}{cc}
-0.1 & -0.35 \\
0 & 0.3
\end{array}\right]
$$

and $\|\rho\| \leqslant 0.035$ [12]. Let $\rho_{m}=0.035$ [13] and set

$$
\begin{aligned}
A_{1} & =\left[\begin{array}{cc}
0 & -0.12+12 \rho_{m} \\
1 & -0.465-\rho_{m}
\end{array}\right], A_{2}=\left[\begin{array}{cc}
0 & -0.12-12 \rho_{m} \\
1 & -0.465+\rho_{m}
\end{array}\right] \\
A_{1 d} & =A_{2 d}=A_{d}=\left[\begin{array}{cc}
-0.1 & -0.35 \\
0 & 0.3
\end{array}\right]
\end{aligned}
$$


When $\mu=0$, the upper bound on the time delay obtained in [13] is 0.863 . However, by Corollary 4 the system $\Sigma_{1}$ is robustly stable for delay $\tau=0.8758$, which is better than the values in [13]. Tab. 1 shows a comparison of the upper bounds for $\mu \neq 0$ obtained by Fridman and Shaked's method [11]-[12], He'method ${ }^{[13]}$ and our methods (Corollary 2). It is clear that the upper bounds obtained by Corollary 2 are larger than those given in $[11,12]$ and [13].

Tab. 1 Calculation results for example 1

\begin{tabular}{|l|l|l|l|l|}
\hline$\mu$ & 0 & 0.1 & 0.5 & 0.9 \\
\hline Fridman $^{[1,12]}$ & 0.782 & 0.736 & 0.465 & 0.454 \\
\hline He [13] & 0.863 & 0.786 & 0.465 & 0.454 \\
\hline Corollary 2 & 0.8758 & 0.8041 & 0.625 & 0.603 \\
\hline
\end{tabular}

Example 2 Consider the robust stability of the uncertain stochastic delay system $\Sigma_{2}$ with the following parameters:

$$
\begin{aligned}
& A=\left[\begin{array}{cc}
-2 & 0.2 \rho \\
1 & -2+0.2 \sigma
\end{array}\right], A_{d}=\left[\begin{array}{cc}
-1 & 0.2 \rho \\
-0.8 & -1.5+0.2 \sigma
\end{array}\right] \\
& C=\left[\begin{array}{cc}
0.1 & -0.1+0.2 \rho \\
0 & 0.1+0.2 \sigma
\end{array}\right], C_{d}=\left[\begin{array}{cc}
-0.3 & 0.2 \rho \\
0 & 0.1+0.2 \sigma
\end{array}\right]
\end{aligned}
$$

and $|\rho| \leqslant 1,|\sigma| \leqslant 1$.

The parameter uncertainty can be represented by a fourvertex polytope, and the upper bound of the time delay $\tau$, which guarantee the given system is exponentially stable in mean square, is given in the Tab. 2 .

Tab. 2 The upper bound of $\tau$ for exponential stability in mean square, as a function of the bound $\mu$

\begin{tabular}{|c|c|c|c|c|c|}
\hline$\mu$ & $0-0.35$ & 0.35 & 0.4 & 0.5 & 0.9 \\
\hline$\tau_{\max }$ & $\rightarrow \infty$ & 2.1538 & 1.4920 & 1.0526 & 0.4926 \\
\hline
\end{tabular}

\section{CONCLUSION}

This paper presents some new stability criteria for stochastic time-varying delay systems with polytopic-type uncertainties. By choosing a new type of Lyapunov-Krasovskii functional based on the equivalent descriptor form of the original system and introducing some free weighing matrices, new technique are developed to make the criteria less conservative. Finally, Numerical examples demonstrate the criteria presented here perform much better than existing stability criteria.

\section{REFERENCES}

[1] HAUSSMAN U G. Asymptotic stability of the linear Itô equation in infinite dimensions[J]. Journal of Mathematical Analysis and Applications, 1978, 65: 219-235.

[2] ICHIKAWA A. Stability of semi-linear stochastic evolution equations[J]. Journal of Mathematical Analysis and Applications, 1982, 90: 12-44.

[3] MAO X, KOROLEVA N, RODKINA A. Robust stability of uncertain stochastic differential delay equations[J]. Systems \& Control Letters, 1998, 35: 325-336.

[4] VERRIEST E I, FLORCHINGER P. Stability of stochastic systems with uncertain time delays[J]. Systems \& Control Letters, 1995, 24: 41-47.

[5] YUE D, WON S. Delay-dependent robust stability of stochastic systems with time delay and nonlinear uncertainties[J]. Electron. Letters, 2001, 37: 992-993.

[6] LU C Y, TSAI J S H. An LMI-Based approach for robust stabilization of uncertain stochastic systems with time-varying delays[J]. IEEE Trans. on Automatic. Control, 2003, 48(2): 286-289.

[7] CHEN W, GUAN Z, LU X. Delay-dependent exponential stability of uncertain stochastic systems with multiple delays: an LMI approach[J]. Systems \& Control Letters, 2005, 54: 547-555.

[8] LU C Y, SU T J, TSAI J S H. On robust stabilization of uncertain stochastic time-delay systems-an LMI-based approach[J]. Journal of the Franklin Institute, 2005, 342: 473487.

[9] FRIDMAN E, SHAKED U. A descriptor system approach to $H_{\infty}$ control of time-delay systems[J]. IEEE Trans. on Automatic Control, 2002, 47: 253-279.

[10] WANG Y, XIE L, De SOUZA C E. Robust control of a class of uncertain nonlinear system[J]. Systems \& Control Letters, 1992, 19: 139-149.

[11] FRIDMAN E, SHAKED U. An improved stabilization method for linear time-delay systems[J]. IEEE Trans. on Automatic Control, 2002, 47: 1931-1937.

[12] FRIDMAN E, SHAKED U. Parameter dependent stability and stabilization uncertain time-delay systems[J]. IEEE Trans. on Automatic Control, 2003, 48: 861-866.

[13] HE Y, WU M, SHE J, LIU G. Parameter-dependent Lyapunov functional for stability of time-delay systems with polytopic-type uncertainties[J]. IEEE Trans. on Automatic Control, 2004, 49(5): 828-832. 